\title{
Local delivery of osteopontin attenuates vascular remodeling by altering matrix metalloproteinase-2 in a rabbit model of aortic injury
}

\author{
Ralf G. Seipelt, MD, ${ }^{a, b}$ Carl L. Backer, MD, ${ }^{a}$ Constantine Mavroudis, MD, ${ }^{a}$ Veronica Stellmach, $\mathrm{PhD}^{\mathrm{c}}$ \\ Mona Cornwell, HT, ${ }^{c}$ Ingrid M. Seipelt, MD, ${ }^{c}$ Friedrich A. Schoendube, MD, and Susan E. Crawford, MD
}

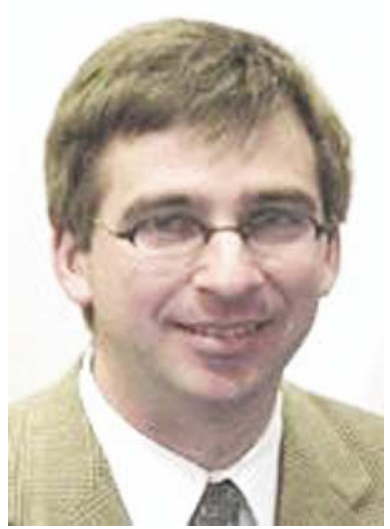

Dr Seipelt
From the Department of Surgery, Division of Pediatric Cardiovascular Thoracic Surgery, Children's Memorial Hospital, Northwestern University Feinberg School of Medicine, ${ }^{\mathrm{a}}$ Chicago, Ill; Department of Thoracic and Cardiovascular Surgery, Georg August University, Göttingen, Germany; and Department of Pathology, Northwestern University Feinberg School of Medicine, ${ }^{\mathrm{c}}$ Chicago, Ill.

This work was supported in part by National Institutes of Health grant CA64329 and Children's Heart Foundation.

Received for publication Oct 21, 2004; revisions received Dec 6, 2004; accepted for publication Dec 17, 2004

Address for reprints: Carl L. Backer, Division of Cardiovascular-Thoracic Surgery, Northwestern University Feinberg School of Medicine, Children's Memorial Hospital, 2300 Children's Plaza, M/C 22, Chicago, IL 60614 (E-mail: cbacker@ childrensmemorial.org)

J Thorac Cardiovasc Surg 2005;130:355-62 $0022-5223 / \$ 30.00$

Copyright (C) 2005 by The American Association for Thoracic Surgery

doi:10.1016/j.jtcvs.2004.12.040
Objective: Vascular remodeling, often accelerated after cardiovascular procedures, may result in stenosis or aneurysm formation. The bone-associated protein osteopontin has been suggested to be involved in vascular remodeling, yet the effect of locally applied osteopontin in an acute vascular injury model of aortic calcification has not been examined.

Methods: Vascular healing of rabbit thoracic aortas treated locally with recombinant osteopontin (dose: $1 \mu \mathrm{g} ; \mathrm{n}=16$ ) or albumin (control, $\mathrm{n}=16$ ) after acute injury created by end-to-end anastomosis was evaluated. Matrix metalloproteinase-2 level was quantified by gelatin zymography. Proliferation of smooth muscle cells was detected by immunostaining for proliferative cell nuclear antigen.

Results: Osteopontin-treated aortas showed significantly diminished vascular remodeling with less calcification $(P=.001)$ and reduced anastomotic luminal stenosis ( $4 \%$ vs $28 \%, P=.002$ ) compared with controls 2 months postsurgery. Moreover, osteopontin-treated aortas revealed a thickened adventitia with increased fibrosis $(P=.006)$. Matrix metalloproteinase-2 level was up-regulated 2-fold with osteopontin treatment compared with control at 1 week, returning to baseline by 1 month. Staining for proliferation cell nuclear antigen disclosed an increase in proliferation cell nuclear antigen-positive smooth muscle cells in the media of osteopontin-treated aortas at 1 week, normalizing by 1 month.

Conclusions: These data suggest a beneficial effect of locally applied osteopontin after acute injury possibly by altering matrix metalloproteinase- 2 activity and smooth muscle cell proliferation. Brief application of osteopontin may effectively enhance vascular healing by reducing calcification and thus maintaining luminal integrity. The role of the osteopontin-related increase in adventitial fibrosis on vascular healing has to be explored.

$\mathrm{V}$ ascular remodeling can result in vascular obstructive disease, ${ }^{1}$ often accompanied by pathologic calcification or vascular aneurysmal disease as observed in bicuspid aortic valve disease and Marfan syndrome. ${ }^{2}$ These structural changes of the arterial wall often develop or are accelerated after cardiovascular surgical or interventional procedures, and therapeutic options to halt this disease are limited. We recently reported that in an experimental rabbit model, which simulates cardiovascular surgical interventions, recombinant vascular endothelial growth factor protected the aorta from pathologic remodeling and calcification, ${ }^{3}$ possibly by up-regulating vascular osteopontin (OPN) levels in the early phase after arterial injury. ${ }^{4}$

OPN, a multifunctional acidic phosphoprotein normally expressed in mineralized tissues such as bone and teeth, as well as in kidney, ${ }^{5}$ is present in uninjured arteries at a low level but is expressed abundantly in injured arteries, ${ }^{4,6}$ suggesting a role in 
vascular remodeling after injury. Moreover, up-regulated levels of OPN have been observed at sites of dystrophic calcification in human atherosclerotic plaques and calcified aortic valves. ${ }^{7-9}$ An in vitro study ${ }^{10}$ using vascular smooth muscle cells (SMCs) and a study ${ }^{11}$ using OPN knockout mice $\left(\mathrm{OPN}^{-/}\right)$implied that the up-regulation of OPN has a protective role to limit vascular calcification. In contrast, other studies support a pathologic role of OPN as an inducer of vascular SMC proliferation and migration, ${ }^{12}$ thus contributing to an increase in medial thickness and neointimal hyperplasia after cuff-induced injury. ${ }^{13}$ Carotid artery ligation in OPN-null mice ${ }^{14}$ and neutralizing antibodies directed against OPN after endothelial denudation diminished neointimal formation. ${ }^{15}$ These apparent opposing actions suggest that context and timing of OPN expression could be important determinants of whether OPN acts as an inducer or inhibitor of vascular remodeling.

Matrix metalloproteinases (MMPs) appear to be intimately involved in vascular remodeling by degrading extracellular matrix, and their activity is up-regulated in a wide spectrum of vascular diseases including aortic aneurysm formation ${ }^{16,17}$ and Kawasaki disease, ${ }^{18}$ as well as atherosclerotic plaque formation ${ }^{19}$ and intimal hyperplasia after acute injury. ${ }^{20,21}$ MMPs can exert several actions on the vessel wall, some of which involve OPN. Stimulation of SMCs with OPN has been shown to increase MMPs, ${ }^{22}$ and aortas of mice overexpressing OPN demonstrate elevated MMP-2 and MMP-9 levels compared with wild-type mice. $^{13}$

In the present study we used our experimental rabbit model, which simulates cardiovascular surgical interventions prone to failure because of accelerated vasculopathy including medial calcification, ${ }^{3}$ to determine the effect of recombinant OPN, locally applied at the time of acute arterial injury, on vascular remodeling. We hypothesized that topically delivered OPN may diminish vascular calcification and thus enhance healing of aortic end-to-end anastomosis with reduced vascular stenosis.

\section{Methods}

\section{Acute Aortic Injury Model}

Thoracic aortic injury was performed on 32 male New Zealand White rabbits ( 9 weeks old, mean weight $2.5 \pm 0.3 \mathrm{~kg}$ ) as previously described. ${ }^{3,4}$ Briefly, the thoracic aorta was dissected free just below the left subclavian artery in anesthetized rabbits. After proximal and distal crossclamping, a 10-mm (confirmed by measurement) ring of thoracic aorta was resected. This was followed by an end-to-end anastomosis with the 7-0 Prolene running suture technique (tension model). After surgery, the rabbits were allowed to recuperate; they were maintained under standardized conditions with a regular day and night cycle (12/12 hours), and free access to water and standard rabbit diets (Hi-Fiber 5P25, Prolab, PMI Nutrition International, St Louis, Mo). All animal experiments were performed with approval of the Animal Care and Use Committee of Children's Memorial Institute of Education and Research and in accordance with the "Guide for the Care and Use of Laboratory Animals" (National Institutes of Health publication No. 85-23, revised 1996).

\section{Osteopontin Treatment}

Treatment consisted of applying either $1 \mu \mathrm{g}$ of recombinant mouse OPN (R\&D Systems, Minneapolis, Minn) or the vehicle phosphatebuffered saline and $0.1 \%$ albumin as control to the intraluminal surface, while performing the anastomosis (10 minutes exposure time), and to the serosal surface ( $1 \mathrm{~mm}$ beyond the crossclamps), for 10 minutes after finishing the anastomosis, by using a 1-mL syringe with a $27 \mathrm{G}$ needle (Sherwood Medical) to drip $0.2 \mathrm{~mL}$ of solution to the intraluminal and serosal surfaces. At no time did the needle make contact with the vessel wall.

\section{Histology and Morphometry}

At postoperative time intervals of 1 day $(n=4), 1$ week $(n=8)$, 1 month $(\mathrm{n}=10)$, and 2 months $(\mathrm{n}=10)$, equal numbers of control and OPN-treated animals were weighed and sacrificed. The aorta was transected longitudinally, the inner circumference was measured at the anastomosis and $1 \mathrm{~cm}$ proximal and distal to the anastomosis, and the internal diameter and luminal area were calculated as described. ${ }^{3}$ The relative area change assessed at the anastomotic site compared with the area $1 \mathrm{~cm}$ proximal to the site was calculated. For histologic examination, the proximal segment of the anastomotic area was divided into 3 equal sections: the anastomosis itself, the portion including the proximal crossclamp, and the aortic wall between the anastomosis and the crossclamp. Parallel sections from each group were assessed, and a mean score was calculated for each aorta using values obtained from all 3 segments. In addition, a segment $1 \mathrm{~cm}$ distal to the anastomosis was examined. The tissue was fixed in $10 \%$ formalin overnight. The aorta adjacent to the anastomotic suture line up to and including the distal crossclamp area was snap-frozen in liquid nitrogen and stored at $-80^{\circ} \mathrm{C}$. The formalin-fixed tissue was processed and embedded in paraffin, and 4- $\mu \mathrm{m}$ sections were placed on a glass slide and stained with hematoxylin-eosin, elastica van Gieson, and Von Kossa stains. The degree of fibrosis and calcification was graded 1 to $4(1=$ none; $4=$ severe $)$ by a pathologist (S.E.C.) blinded to treatment regimen as previously described. ${ }^{3,4}$

Medial thickness and thickness of adventitial fibrosis were measured at 4 positions on each of 3 histologic sections per aorta. The first position was randomly chosen, and the others at approximately $45^{\circ}, 90^{\circ}$, and $135^{\circ}$ around the vessel perimeter ${ }^{23}$; the average of the 12 measurements was taken per animal as the mean thickness. The external aortic diameter, with the border of adventitial fibrosis-surrounding tissue as the outer limit, was calculated at the anastomosis and $1 \mathrm{~cm}$ proximal and distal to the anastomosis.

\section{Immunohistochemistry}

Paraffin-embedded aortic sections were immunostained using an antibody directed against proliferating cell nuclear antigen (PCNA; Dako, Carpinteria, Calif). Sections were dried at $42^{\circ} \mathrm{C}$ for 24 hours, deparaffinized, incubated at room temperature with the primary antibody, incubated with an avidin-biotin peroxidase conjugated secondary antibody, and counterstained with hematoxylin. Those cells exhibiting a brown color were scored positive. At least 


\section{Control}
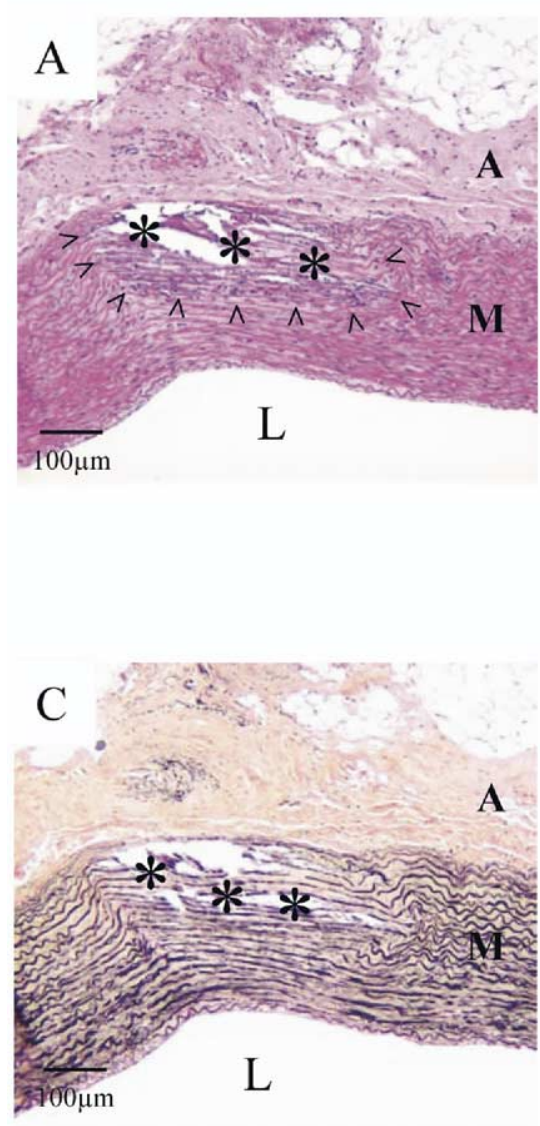

\section{OPN-treated}
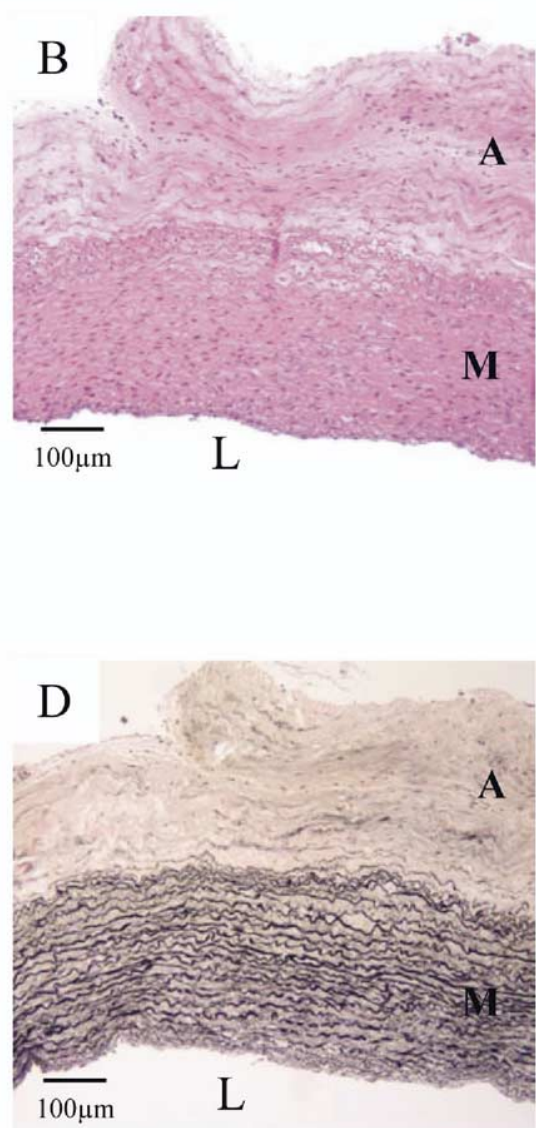

Figure 1. Local recombinant OPN altered aortic healing after end-to-end anastomosis with significantly diminished calcification but increased adventitial fibrosis. Aortic sections were adjacent sections harvested 2 months postinjury. A and B, Hematoxylin-eosin staining. Controls revealed a region of dystrophic calcification (*), whereas OPN-treated aortas had minimal medial pathology. Interface between calcific deposit and media (open arrowheads). The dystrophic calcification disrupted the integrity of the vascular media. Note the increase in adventitial fibrosis of OPN-treated aortas. C and D, Elastica van Gieson staining. Comparable medial thickness was seen in control and OPN-treated aortas. Note alteration in elastica at site of calcific deposit within the media. OPN, Osteopontin; $A$, adventitia; $M$, media; $L$, lumen.

1000 SMCs were counted in each aorta, and the percentage of SMCs with positive PCNA staining was calculated.

\section{Gelatinase Zymography}

Collagenase IV (gelatinase) activity in both the control and OPNtreated thoracic aortas was determined by zymography with $10 \%$ sodium dodecyl sulfate-polyacrylamide gel electrophoresis containing $0.1 \%$ gelatin $(1 \mathrm{mg} / \mathrm{mL}$, Sigma, St Louis, Mo) as described elsewhere. ${ }^{17,19}$ Briefly, the snap-frozen aortic tissue was homogenized in radioimmunoprecipitation (RIPA) buffer, and the protein concentration was determined by protein assay (Bio-Rad, Hercules, Calif). Equal amounts of protein $(30 \mu \mathrm{g})$ were mixed with sample buffer and electrophoresed under nonreducing conditions. After electrophoresis, sodium dodecyl sulfate was eluted from the gel in $2.5 \%$ Triton $\mathrm{X}-100$ for 30 minutes at room temperature. The gels then were incubated in $50 \mathrm{mmol} / \mathrm{L}$ Tris- $\mathrm{HCl}, \mathrm{pH} 8.0,0.2$ $\mathrm{mol} / \mathrm{L} \mathrm{NaCl}, 5 \mathrm{mmol} / \mathrm{L} \mathrm{CaCl}_{2}$, and $0.02 \%$ Brij 35 at $37^{\circ} \mathrm{C}$ for 16 hours. After the gel was stained with Coomassie blue R-250, gelatin-degrading enzymes were identified by their ability to clear the substrate at their respective molecular weights. Gels were calibrated with molecular weight markers (Bio-Rad). Each sample was quantified by densitometric methodology.

\section{Statistical Analysis}

Results are expressed as mean \pm SEM. Statistical differences between nominally and ordinally scaled variables were determined with the chi-square test. Continuous variables were analyzed with the unpaired 2-tailed Student $t$ test and analysis of variance. 
TABLE 1. Difference in vascular medial calcification and adventitial fibrosis between control ( $n=15$ histologic sections; three sections/aorta) and osteopontin-treated ( $n=15$ ) aortic specimens two months postinjury (medial calcification $P<.001$; adventitial fibrosis $P=.004$ )

\begin{tabular}{lcc}
\hline Variable & Control & OPN treated \\
\hline Medial calcification & & \\
$\quad$ None/trace & 0 & 11 \\
Mild & 3 & 2 \\
$\quad$ Moderate & 3 & 2 \\
$\quad$ Severe & 9 & 0 \\
Adventitial fibrosis & & \\
$\quad$ None/trace & 0 & 0 \\
Mild & 5 & 0 \\
Moderate & 10 & 9 \\
Severe & 0 & 6 \\
\hline
\end{tabular}

OPN, Osteopontin.

\section{Results}

\section{Effect of OPN on Vascular Calcification}

Control and OPN-treated rabbits had similar postoperative courses with comparable percentage gain in total body weight 2 months after surgery. Histologically, the control aortas revealed marked remodeling within 1 week, and these pathologic findings progressed with time. By 1 and 2 months postopertively (Figure 1, $A, C$ ), all controls showed band-like regions of dystrophic medial calcification determined by von Kossa staining (data not shown). In striking contrast, OPN treatment resulted in a distinct pattern of vascular remodeling at all time points. At 2 months postoperatively, medial calcification was significantly reduced compared with controls $(P<.001$, Table 1$)$, and in 3 of 5 OPN-treated aortic specimens islands of calcification were absent in all 3 sections taken for histology (Figure 1, $B, D$ ). In 2 aortic specimens, there was focal calcification at the suture line only.

Adventitial Fibrosis and Anastomotic Luminal Stenosis An unexpected finding of our experiments was the significant increase in adventitial fibrosis in OPN-treated aortas

TABLE 2. Morphologic features of the aortic wall two months after acute injury

\begin{tabular}{lccc}
\hline & $\begin{array}{c}\text { Control } \\
(\mathbf{n}=\mathbf{5})\end{array}$ & $\begin{array}{c}\text { OPN treated } \\
\text { (n = 5) }\end{array}$ & $\begin{array}{c}\boldsymbol{P} \\
\text { value }\end{array}$ \\
\hline $\begin{array}{lccc}\text { Luminal area stenosis }(\%) \\
\text { Medial thickness }(\mu \mathrm{m})\end{array}$ & $28.2 \pm 4.9$ & $4.1 \pm 1.7$ & .002 \\
$\begin{array}{l}\text { Thickness of adventitial } \\
\quad \text { fibrosis }(\mu \mathrm{m})\end{array}$ & $148 \pm 16$ & $307 \pm 14$ & .31 \\
$\quad$ Vessel wall thickness $(\mu \mathrm{m})$ & $428 \pm 14$ & $246 \pm 17$ & .001 \\
& & & \\
\end{tabular}

OPN, Osteopontin. compared with controls $(P=.004$, Table 1$)$. The thickness of adventitial fibrosis increased in OPN-treated aortas compared with controls by 1 week, and these morphologic differences became even more apparent by 2 months postoperatively (Table 2, Figure 1, A, B). However, this morphologic change did not impinge on the vascular lumina. By 2 months postoperatively, the internal diameter at the anastomosis with OPN treatment was significantly greater compared with controls (mean internal diameter: $4.3 \pm 0.3 \mathrm{~mm}$ in OPN-treated aorta vs $3.7 \pm 0.4 \mathrm{~mm}, P=.03$ ). OPN treatment significantly diminished the luminal stenosis at the anastomotic site to only $4 \%$, whereas the control group specimens revealed a luminal stenosis of $28 \%(P=.002$, Table 2). Furthermore, the control aortas revealed a significantly diminished external diameter at the anastomosis compared with $1 \mathrm{~cm}$ proximal because of the reduced internal diameter, and thus luminal area (Table 3).

\section{Matrix Metalloproteinases}

A gelatinolytic band at $72 \mathrm{kDa}$, corresponding to the latent form of MMP-2, was present in both groups at all time points (Figure 2, A). Densitometric quantification indicated an increase in latent MMP-2 in OPN-treated aortas compared with controls at 1 week, returning to baseline by 1 month. A second gelatinolytic band at $62 \mathrm{kDa}$ revealed the activated form of MMP-2. In both groups, there was a rapid increase in activated MMP-2 by 24 hours, peaking at 1 week. OPN induced a transient increase in MMP-2 revealing a 1.9-fold up-regulation in activated MMP-2 compared with control at 1 week $(P<.001)$ and a return to time 0 levels by 1 month, whereas activated MMP-2 was more chronically elevated with a persistent increase in the control aortas (Figure 2, $A, B$ ). At 24 hours, lesser intensity bands at 92 and $80 \mathrm{kDa}$ (most likely representing pro-MMP-9 and its activated form) ${ }^{24}$ appeared in the OPN treatment group, whereas the control group revealed a pro-MMP-9 band only. MMP-9 levels returned to baseline by 1 week in both groups.

\section{SMC Proliferation}

Because OPN is known to promote vascular SMC proliferation in vitro ${ }^{12,25}$ and in mice overexpressing $\mathrm{OPN},{ }^{13}$ we determined whether there was an alteration of medial SMC proliferation after brief exposure to OPN in our model. The pattern of SMC proliferation was different between the 2 groups. By 1 week postoperatively, the OPN-treated aortic media showed a significant increase in PCNA-positive SMC nuclei (Figure 3, B, inset), in contrast with the only rare positive staining SMC nucleus in the control aortas (Figure 3, A). By 1 month, the SMC proliferation in OPN-treated aortas diminished to levels seen in control aortas (Figure 3,C). Although there appeared to be an OPN-mediated increase in SMC proliferation when the medial thickness was measured, the control and OPN-treated 
TABLE 3. External diameter of the proximal aorta, anastomosis, and distal aorta two months postinjury

\begin{tabular}{llccc}
\hline Variable & Segment & Control $(\mathbf{n}=\mathbf{5})$ & OPN treated $(\mathbf{n}=\mathbf{5})$ & \multicolumn{1}{c}{$\boldsymbol{P}$ value } \\
\hline & Proximal aorta & $5.47 \pm 0.18$ & $5.60 \pm 0.046$ & .543 \\
External diameter $(\mathrm{mm})$ & Anastomosis & $4.77 \pm 0.28$ & $5.65 \pm 0.10$ & .034 \\
& Distal aorta & $5.5 \pm 0.13$ & $5.54 \pm 0.06$ & .741 \\
\hline
\end{tabular}

OPN, Osteopontin.

aortic media were comparable 2 months postoperatively (Table 2). Moreover, the mean number of elastic lamellae was similar in both groups when avoiding the band-like regions of calcification in the control aortas (Figure 1, C, D; mean number of elastic lamellae $20.9 \pm 0.6$ in controls vs $21.4 \pm 0.5$ in OPN-treated aortas, respectively). In these regions of the control aortas, elastic lamellae were replaced by calcification and thus reduced. Intimal proliferative lesions were not observed in the control or the OPN-treated aortas at any time point.

\section{Discussion}

To our knowledge, this is the first in vivo study to show that a single dose of recombinant OPN applied at the site of an acute arterial injury is capable of effectively suppressing calcification and modulating vascular remodeling. Arterial obstructive disease, often associated with pathologic calcification, and vascular aneurysm disease are the result of severe vascular remodeling contributing to a high rate of morbidity and mortality. Recently, OPN, a bone-associated
A

Control

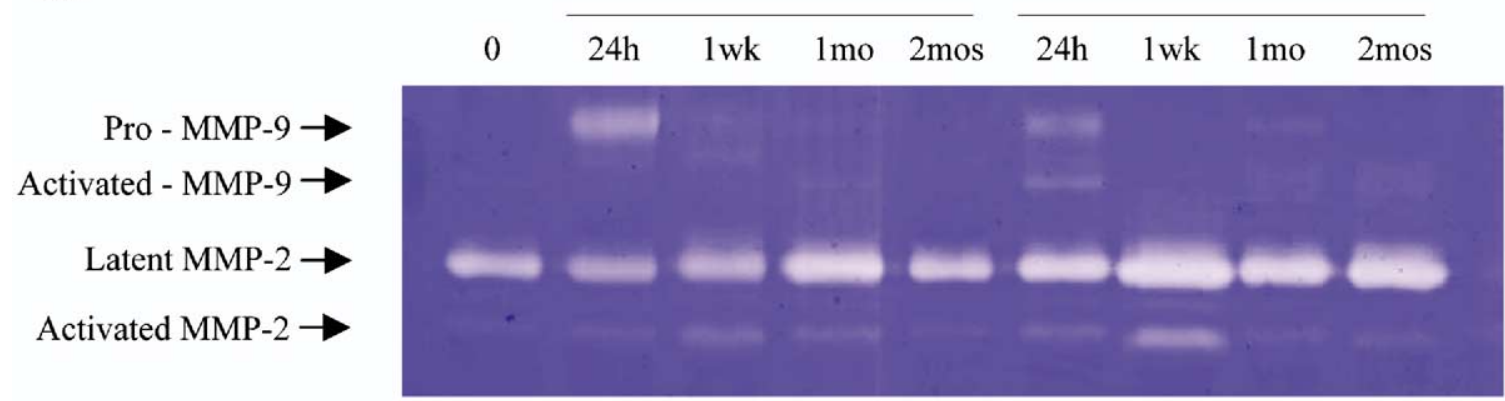

B

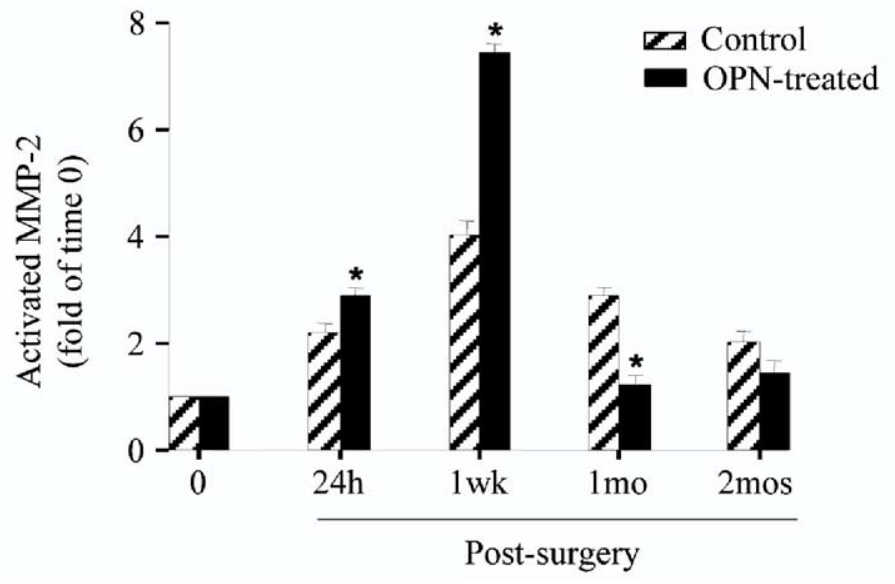

Figure 2. Representative zymograms for pro-MMP-2 and activated MMP-2 and pro-MMP-9 and activated MMP-9 showing increased MMP-2 level at 1 week in OPN-treated aorta (right, A) when compared with controls (left, A). $B$, corresponding densitometric analysis of the activated MMP-2. Results are mean \pm SEM. ${ }^{*} \boldsymbol{P}<.05$ compared with control group. OPN, Osteopontin; MMP, matrix metalloproteinase; 0 , level at time 0 . 

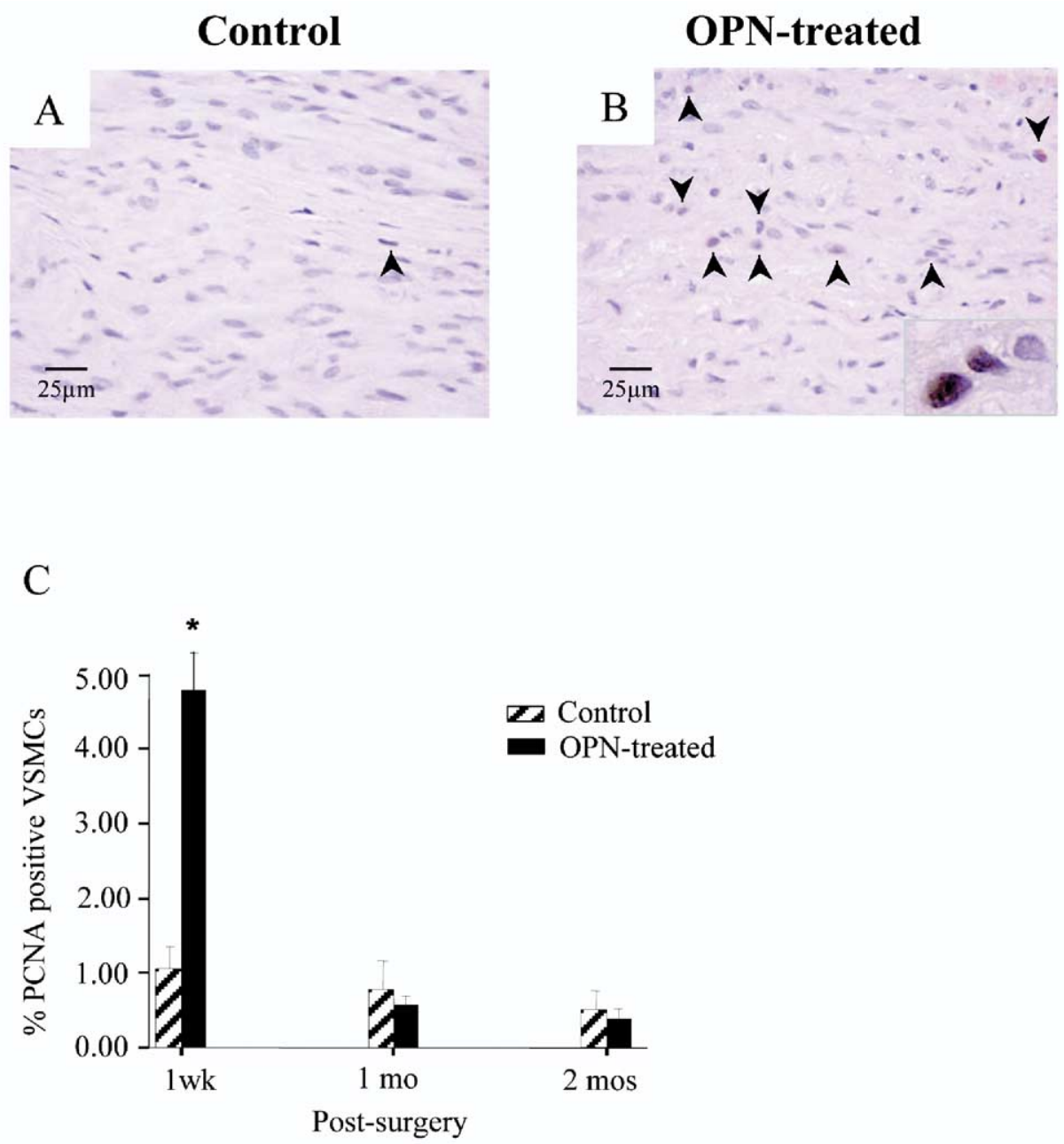

Figure 3. Immunohistochemical staining for PCNA of control (A) and OPN-treated aortas (B) harvested 1 week postinjury revealed increased vascular smooth muscle cell (SMC) proliferation after OPN treatment. Positive nuclei (arrowheads). Inset in (B) demonstrates $2 \mathrm{PCNA}^{+}$nuclei next to 1 negative nucleus. C, OPN treatment resulted in a significant increase in the percentage of cells with positive PCNA staining by 1 week. Results are mean \pm SEM. ${ }^{*} P<.05$ compared with control group. OPN, osteopontin; PCNA, proliferating cell nuclear antigen; VSMC, vascular smooth muscle cell.

protein, has been implicated in vascular remodeling. Although OPN-deficient mice showed no disturbance in vascular calcification when crossed with matrix Gla proteinnull mice ${ }^{26}$ or Apo-E-null mice, ${ }^{27}$ they had increased levels of arterial calcification. A hemodynamic study in OPN-null mice demonstrated greater vascular wall compliance, suggesting a regulatory role in normal vascular physiology. ${ }^{14}$ However, studies in OPN-overexpressing mice suggested OPN to be involved in the development of atherosclerosis and vascular stenosis because of intimal proliferation. ${ }^{13}$ These diverse actions of OPN on the vascular wall make it difficult to predict whether a single dose of OPN, exogenously delivered, has a beneficial or adverse effect on vascular healing after acute injury. To more directly assess a potential therapeutic role of OPN on vascular remodeling, and in particular on vascular calcification, we applied recombinant OPN locally at the site of aortic injury in an acute arterial injury model. ${ }^{3}$ In this model we performed a similar operation as in coarctation repair with excision of an aortic ring and end-to-end anastomosis; however, the aortic ring in coarctation repair is mainly ductal tissue. Our in vivo data revealed that a single dose of OPN locally applied at the time of arterial injury is capable of maintaining the vessel lumen possibly by suppressing the development of pathologic calcification. An unexpected finding occurred within the adventitial segment of the vessel. Adventitial 
fibrosis, determined by histologic grading and measurements, was found to be significantly increased in OPNtreated aortas compared with controls. Recent data suggest that OPN may play a role in the development of tissue fibrosis, because OPN is chemotactic for fibroblasts and potentiates growth factor-mediated fibroblast proliferation. ${ }^{28,29}$ In the present study it is unclear whether the OPN-related increase in adventitial fibrosis might be beneficial to vessels prone to aneurysmal formation or might alter the compliance of the vessel, resulting in enhanced wall stress and early degeneration. Therefore, further studies with longer time intervals and a model of developing aortic aneurysm are needed.

Our results indicate 2 other potential mechanisms underlying the ability of OPN to alter vascular remodeling when exogenously applied at the time of injury. The first observation is related to rapid but transient up-regulation of MMP-2 and activated MMP-9, and the second observation involves transient increase of SMC proliferation. MMP-2, a $72 \mathrm{kDa}$ type IV collagenase that degrades extracellular matrix and basement membrane proteins (eg, collagen and elastin) and that enhances SMC migration, is secreted from a variety of cell types, including vascular endothelial cells and SMCs. ${ }^{30}$ Several studies suggest that MMP-2 and MMP-9 are involved in vascular remodeling associated with vascular disease and injury, resulting in arterial obstructive disease or aneurysm formation. ${ }^{16-21}$ Our data confirm the involvement of MMP-2 and MMP-9 in vascular remodeling because both were increased after arterial injury. Brief exposure to OPN in this study was a trigger to more rapidly but transiently up-regulate MMP-2 activity 2 -fold compared with control aortas with return to time 0 levels by 1 month. The transient increase in MMP-2 may be beneficial in this model to facilitate replacement of dead cell bodies that occur after arterial injury within the media. This would be consistent with the temporary increase in SMC proliferation within the media of OPN-treated aortas. The role of elevated MMP-2 at later time points in control aortas that develop severe vascular remodeling needs to be clarified.

OPN treatment of the aorta has been shown to promote SMC proliferation in vitro. ${ }^{12,25}$ A transgenic mice model with chronic OPN overexpression developed a thickened arterial media, and, after cuff-induced injury, intimal proliferation was noted. ${ }^{13}$ In the current study, the brief exposure of OPN induced an early increase in SMC proliferation; however, this was only a transient activity because the number of PCNA-positive SMCs returned to baseline by 1 month. The medial thickness remained unchanged when compared with control animals, and intimal proliferative lesions were not observed. These results suggest that the short exposure time to OPN after arterial injury may be crucial for its beneficial action, whereas chronic exposure to OPN appears to be associated with deleterious consequences. ${ }^{13}$

\section{Limitations}

This study was performed to determine a possible role of exogenously added OPN on vascular healing as suggested by a previous study performed in our laboratory. ${ }^{4}$ However, at this point we do not know the ideal OPN concentration to promote the best vascular healing. Therefore, the optimal OPN concentration and exposure time will need to be defined in future experiments. Furthermore, we do not know whether intraluminal or adventitial exposure alone is more or less effective on the reduction of medial calcification or adventitial fibrosis. Second, this study only measured isolated metalloproteinase levels ex vivo with zymography. However, the balance of MMPs and their natural inhibitors are important to determine their activity.

In summary, the present study demonstrated that a brief local OPN exposure might be effective to induce a response within the injured arterial wall. OPN was capable of protecting the arterial wall from pathologic vascular remodeling probably by, directly or indirectly, reducing calcification, rapidly up-regulating MMP-2, and increasing SMC proliferation. The role of the OPN-related increase in adventitial fibrosis on vascular healing has to be explored.

\section{References}

1. Birnbaum Y, Fishbein MC, Luo H, Nishioka T, Siegel RJ. Regional remodeling of atherosclerotic arteries: a major determinant of clinical manifestations of disease. J Am Coll Cardiol. 1997;30:1149-64.

2. Niwa K, Perloff JK, Bhuta SM, Laks H, Drinkwater DC, Child JS, et al. Structural abnormalities of great arterial walls in congenital heart disease: light and electron microscopic analyses. Circulation. 2001; 103:393-400.

3. Seipelt RG, Backer CL, Mavroudis CL, Stellmach V, Seipelt IM, Cornwell M, et al. Topical VEGF enhances healing of thoracic aortic anastomosis for coarctation in a rabbit model. Circulation. 2003;108: III50-4.

4. Seipelt RG, Backer CL, Mavroudis CL, Stellmach V, Cornwell M, Seipelt IM, et al. Osteopontin expression and adventitial angiogenesis induced by local VEGF $_{165}$ reduces experimental aortic calcification. J Thorac Cardiovasc Surg. 2005;129:773-81.

5. Mazzali M, Kipari T, Ophascharoensuk V, Wesson JA, Johnson R, Hughes J. Osteopontin — a molecule for all seasons. QJM. 2002;95:3-13.

6. Giachelli CM, Bae N, Almeida M, Denhardt DT, Alpers CE, Schwartz SM. Osteopontin is elevated during neointima formation in rat arteries and is a novel component of human atherosclerotic plaques. $J$ Clin Invest. 1993;92:1686-96.

7. Fitzpatrick LA, Severson A, Edwards WD, Ingram RT. Diffuse calcification in human coronary arteries. Association of osteopontin with atherosclerosis. J Clin Invest. 1994;94:1597-604.

8. O'Brien ER, Garvin MR, Stewart DK, Hinohara T, Simpson JB, Schwartz $\mathrm{SM}$, et al. Osteopontin is synthesized by macrophage, smooth muscle, and endothelial cells in primary and restenotic human coronary atherosclerotic plaques. Arterioscler Thromb. 1994;14:1648-56.

9. Mohler ER 3rd, Adam LP, McClelland P, Graham L, Hathaway DR. Detection of osteopontin in calcified human aortic valves. Arterioscler Thromb Vasc Biol. 1997; 17:547-52.

10. Wada T, McKee MD, Steitz S, Giachelli CM. Calcification of vascular smooth muscle cell cultures: inhibition by osteopontin. Circ Res. 1999;84:166-78.

11. Steitz SA, Speer MY, McKee MD, Liaw L, Almeida M, Yang H, et al. Osteopontin inhibits mineral deposition and promotes regression of ectopic calcification. Am J Pathol. 2002;161:2035-46.

12. Panda D, Kundu GC, Lee BI, Peri A, Fohl D, Chackalaparampil I, et al. Potential roles of osteopontin and alphaVbeta3 integrin in the 
development of coronary artery restenosis after angioplasty. Proc Natl Acad Sci U S A. 1997;94:9308-13.

13. Isoda K, Nishikawa K, Kamezawa Y, Yoshida M, Kusuhara M, Moroi $\mathrm{M}$, et al. Osteopontin plays an important role in the development of medial thickening and neointimal formation. Circ Res. 2002;91:77-82.

14. Myers DL, Harmon KJ, Lindner V, Liaw L. Alterations of arterial physiology in osteopontin-null mice. Arterioscler Thromb Vasc Biol. 2003;23:1021-8.

15. Liaw L, Lombardi DM, Almeida MM, Schwartz SM, deBlois D, Giachelli CM. Neutralizing antibodies directed against osteopontin inhibit rat carotid neointimal thickening after endothelial denudation. Arterioscler Thromb Vasc Biol. 1997;17:188-93.

16. Goodall S, Crowther M, Hemingway DM, Bell PR, Thompson MM. Ubiquitous elevation of matrix metalloproteinase-2 expression in the vasculature of patients with abdominal aneurysms. Circulation. 2001; 104:304-9.

17. Fedak PW, de Sa MP, Verma S, Nili N, Kazemian P, Butany J, et al. Vascular matrix remodeling in patients with bicuspid aortic valve malformations: implications for aortic dilatation. J Thorac Cardiovasc Surg. 2003;126:797-806.

18. Gavin PJ, Crawford SE, Shulman ST, Garcia FL, Rowley AH. Systemic arterial expression of matrix metalloproteinases 2 and 9 in acute Kawasaki disease. Arterioscler Thromb Vasc Biol. 2003;23:576-81.

19. Li Z, Li L, Zielke R, Cheng L, Xiao R, Crow MT, et al. Increased expression of 72-kd type IV collagenase (MMP-2) in human aortic atherosclerotic lesions. Am J Pathol. 1996;148:121-8.

20. Strauss BH, Chisholm RJ, Keeley FW, Gotlieb AI, Logan RA, Armstrong PW. Extracellular matrix remodeling after balloon angioplasty injury in a rabbit model of restenosis. Circ Res. 1994;75: 650-8.

21. Galis ZS, Khatri JJ. Matrix metalloproteinases in vascular remodeling and atherogenesis: the good, the bad, and the ugly. Circ Res. 2002; 90:251-62.
22. Bendeck MP, Irvin C, Reidy M, Smith L, Mulholland D, Horton M, et al. Smooth muscle cell matrix metalloproteinase production is stimulated via alpha(v)beta(3) integrin. Arterioscler Thromb Vasc Biol. 2000;20:1467-72.

23. Stefanadis C, Vlachopoulos C, Karayannacos P, Boudoulas H, Stratos $\mathrm{C}$, Filippides T, et al. Effect of vasa vasorum flow on structure and function of the aorta in experimental animals. Circulation. 1995;91: 2669-78.

24. Southgate KM, Davies M, Booth RF, Newby AC. Involvement of extracellular-matrix-degrading metalloproteinases in rabbit aortic smooth-muscle cell proliferation. Biochem J. 1992;288:93-9.

25. Gadeau AP, Campan M, Millet D, Candresse T, Desgranges C. Osteopontin overexpression is associated with arterial smooth muscle cell proliferation in vitro. Arterioscler Thromb. 1993;13:120-5.

26. Speer MY, McKee MD, Guldberg RE, Liaw L, Yang HY, Tung E, et al. Inactivation of the osteopontin gene enhances vascular calcification of matrix Gla protein-deficient mice: evidence for osteopontin as an inducible inhibitor of vascular calcification in vivo. J Exp Med. 2002;196:1047-55.

27. Matsui Y, Rittling SR, Okamoto H, Inobe M, Jia N, Shimizu T, et al. Osteopontin deficiency attenuates atherosclerosis in female apolipoprotein e-deficient mice. Arterioscler Thromb Vasc Biol. 2003;23: 1029-34.

28. Li G, Oparil S, Kelpke SS, Chen YF, Thompson JA. Fibroblast growth factor receptor-1 signaling induces osteopontin expression and vascular smooth muscle cell-dependent adventitial fibroblast migration in vitro. Circulation. 2002;106:854-9.

29. Takahashi F, Takahashi K, Okazaki T, Maeda K, Ienaga H, Maeda M, et al. Role of osteopontin in the pathogenesis of bleomycin-induced pulmonary fibrosis. Am J Respir Cell Mol Biol. 2001;24:264-71.

30. Galis ZS, Muszynski M, Sukhova GK, Simon-Morrissey E, Unemori EN, Lark MW, et al. Cytokine-stimulated human vascular smooth muscle cells synthesize a complement of enzymes required for extracellular matrix digestion. Circ Res. 1994;75:181-9. 